\title{
The Most Common Cause of Lower Gastrointestinal Bleeding without Other Symptoms in Children is Colonic Polyp: Is Total Colonoscopy Needed?
}

\author{
Yeoun Joo Lee and Jae Hong Park \\ Department of Pediatrics, Pusan National University Children's Hospital, Pusan National University, Yangsan, Korea
}

See "Endoscopic Findings in Children with Isolated Lower Gastrointestinal Bleeding" by Ari Silbermintz, Manar Matar, Amit Assa, et al., on page 258-261.

The incidence of pediatric inflammatory bowel disease is increasing worldwide, with a corresponding increase in the demand for colonoscopy. Colonoscopy is an invasive procedure and can be challenging in children. Additionally, colon cancer is rare in children; therefore, colonoscopy is usually not necessary. Careful history-taking and physical examination can accurately distinguish between several diseases, thereby obviating the need for colonoscopy to establish the diagnosis in children. A child's age is an important factor in narrowing the differential diagnosis. Nevertheless, if diagnoses are not clinically distinguishable, endoscopy is necessary. The European Society for Paediatric Gastroenterology Hepatology and Nutrition guidelines published in 2017 provide indications for colonoscopy in children with unexplained anemia and/ or diarrhea, perianal lesions, rectal bleeding, and unexplained growth retardation. ${ }^{1}$

Although some cases of lower gastrointestinal bleeding can be diagnosed clinically, ${ }^{2}$ diagnosis is challenging in children with lower gastrointestinal bleeding without other symptoms.

Received: April 12, 2019 Revised: May 8, 2019

Accepted: May 8, 2019

Correspondence: Yeoun Joo Lee

Department of Pediatrics, Pusan National University Children's Hospital, Pusan National University, 20 Geumo-ro, Mulgeum-eup, Yangsan 50612, Korea Tel: +82-55-360-2180, Fax: +82-55-360-2181, E-mail: moonmissing@gmail.com ORCID: https://orcid.org/0000-0001-8012-5433

cc This is an Open Access article distributed under the terms of the Creative Commons Attribution Non-Commercial License (http://creativecommons.org/ licenses/by-nc/3.0) which permits unrestricted non-commercial use, distribution, and reproduction in any medium, provided the original work is properly cited.
Silbermintz et al. ${ }^{3}$ described the role of endoscopic examination for diagnosis in children with lower gastrointestinal bleeding without other symptoms. The most common cause of asymptomatic lower gastrointestinal bleeding in children is juvenile polyps; ${ }^{2,4}$ therefore, suspected juvenile polyps in children serve as an indication for colonoscopy. Several previous studies have reported that polyps were found in $7 \%-27.2 \%$ of children undergoing colonoscopy. ${ }^{4-7}$ A multicenter study investigating 13,115 children who underwent colonoscopy in the USA reported that polyps were detected in $6.1 \%$ of all children who underwent colonoscopy and in $12 \%$ of children with lower gastrointestinal bleeding. ${ }^{5}$ A study by Silbermintz et al. $^{3}$ excluded children with clinically suspected inflammatory bowel disease, those with previously diagnosed polyps, and those with familial adenomatous polyposis. Therefore, it is not possible to directly compare the prevalence of colon polyps with that reported in other studies. Nevertheless, Silbermintz et al. ${ }^{3}$ reported that $41.6 \%$ of children who underwent colonoscopy for lower gastrointestinal bleeding without other symptoms were diagnosed with colonic polyps. This indicates that although colonoscopy is challenging in children, this examination is needed because of the relatively high incidence of polyps in children with lower gastrointestinal bleeding without other symptoms. It is important to note that polyps can readily be diagnosed and treated with colonoscopy.

Solitary polyps found in children are known to occur most commonly in the left-sided colon. Silbermintz et al. ${ }^{3}$ reported that left-sided colon polyps were detected in $94.2 \%$ 
of children; however, children with polyposis syndrome or familial adenomatous polyposis were excluded from the study. Therefore, the prevalence of left-sided colon polyps in this study was higher than that observed in other studies. Our previous report demonstrated $84.9 \%$ of polyp located in left-sided colon. ${ }^{8}$ A study that investigated $45 \%$ of children with multiple colon polyps reported that $35 \%$ of all polyps were found proximal to the sigmoid colon. ${ }^{9}$ Another study performed in Taiwan reported that only $43.6 \%$ of juvenile polyps occurred in the rectosigmoid colon, and a solitary polyp was reported in $92.3 \%$ of cases. ${ }^{10}$ Clinicians need to be aware that polyps may occur in the proximal colon and that multiple polyps or polyposis may occur anywhere in the colon; therefore, the initial colonoscopy should include a thorough examination of the entire colon.

In conclusion, colonoscopy is a useful test in children with suspected colonic lesions. The incidence of colonic polyps is relatively high in children with lower gastrointestinal bleeding without other symptoms. Colonoscopy is a useful diagnostic and therapeutic modality in children with colonic polyps. It should be performed in children only when absolutely necessary. Although most colonic polyps tend to occur in the left-sided colon, examination of the entire colon is recommended for accurate diagnosis.

\section{Conflicts of Interest}

The authors have no financial conflicts of interest.

\section{REFERENCES}

1. Thomson M, Tringali A, Dumonceau JM, et al. Paediatric gastrointestinal endoscopy: European Society for Paediatric Gastroenterology Hepatology and Nutrition and European Society of Gastrointestinal Endoscopy guidelines. J Pediatr Gastroenterol Nutr 2017;64:133-153.

2. Sahn B, Bitton S. Lower gastrointestinal bleeding in children. Gastrointest Endosc Clin N Am 2016;26:75-98.

3. Silbermintz A, Matar M, Assa A, Zevit N, Glassberg YM, Shamir R. Endoscopic findings in children with isolated lower gastrointestinal bleeding. Clin Endosc 2019;52:258-261.

4. Park JH. Pediatric colonoscopy: the changing patterns and single institutional experience over a decade. Clin Endosc 2018;51:137-141.

5. Thakkar K, Alsarraj A, Fong E, Holub JL, Gilger MA, El Serag HB. Prevalence of colorectal polyps in pediatric colonoscopy. Dig Dis Sci 2012;57:1050-1055

6. Chen J, Yu H, Zhong W, et al. [Pediatric colonoscopy in South China: a single-center experience from 229 cases]. Zhonghua Wei Chang Wai Ke Za Zhi 2017;20:1404-1408.

7. Yoshioka S, Takedatsu H, Fukunaga S, et al. Study to determine guidelines for pediatric colonoscopy. World J Gastroenterol 2017;23:57735779.

8. Lee BG, Shin SH, Lee YA, Wi JH, Lee YJ, Park JH. Juvenile polyp and colonoscopic polypectomy in childhood. Pediatr Gastroenterol Hepatol Nutr 2012;15:250-255.

9. Lehmann CU, Elitsur Y. Juvenile polyps and their distribution in pediatric patients with gastrointestinal bleeding. W V Med J 1996;92:133-135.

10. Wu CT, Chen CA, Yang YJ. Characteristics and diagnostic yield of pediatric colonoscopy in Taiwan. Pediatr Neonatol 2015;56:334-338. 